九州大学学術情報リポジトリ

Kyushu University Institutional Repository

\title{
Direct measurement procedure for three- dimensional local crack driving force using synchrotron X-ray microtomography
}

Toda, Hiroyuki

Faculty of Engineering, Kyushu University : Professor

Yamamoto, S.

Department of Production Systems Engineering, Toyohashi University of Technology

小林，正和

豊橋技術科学大学

Uesugi, kentaro

Japan Synchrotron Radiation Research Institute

他

http://hdl. handle. net/2324/1807816

出版情報: Acta materialia. 56（20)，pp.6027-6039，2008-12-01. Elsevier バージョン：

権利関係 : 


\title{
Direct measurement procedure for three-dimensional local crack
}

\section{driving force using synchrotron X-ray microtomography}

\author{
H. Toda ${ }^{a}$, S. Yamamoto ${ }^{a}$, M. Kobayashi ${ }^{\text {a }}$ K. Uesugi ${ }^{b}$ and H. Zhang ${ }^{a}$ \\ ${ }^{a}$ Department of Production Systems Engineering, Toyohashi University of Technology, Toyohashi, \\ Aichi 441-8580, Japan (e-mail: toda@pse.tut.ac.jp, tel: +81-532446697, fax: +81-532446690 (H. \\ Toda) \\ b Japan Synchrotron Radiation Research Institute, 1-1-1, Kouto, Mikazuki-cho, Sayo-gun, Hyogo \\ 679-5198 Japan
}

\begin{abstract}
X-ray microtomography has been utilized for the observation of ductile fractures in an aluminum alloy with an Al/Al-Si dual phase structure. A procedure for analyzing a series of tomographic images is proposed for extracting the variation in the local crack-tip opening displacement (CTOD), and its feasibility is confirmed. Complicated crack growth behavior and the formation of uncracked ligaments ahead of a crack tip are observed in the alloy owing to the marked difference in local fracture toughness between the two phases. The proposed technique has provided a quantitative interpretation for such phenomena. It is clarified that a conventional measurement significantly overestimates the CTOD level. The transition behavior in CTOD has been revealed over a certain distance across an interface between the two phases, suggesting the existence of scaling effects that influence the microstructure/fracture relationship. Overall the current method could offer a highly effective way of assessing 3-D local fracture behavior.
\end{abstract}

Keywords: Fracture; Cast Aluminum Alloys; Image Analysis; Local Crack Driving Force; Synchrotron Radiation 


\section{Introduction and Background}

Numerous experimental reports have been devoted to measuring crack driving forces under monotonic or cyclic loading. The techniques can be broadly classified as optical methods, such as photoelasticity [1] and moiré interferometry [2], surface-based microscopy methods, such as in-situ stereoimaging with scanning electron microscopes [3], and a few limited methods such as direct strain measurement using embedded optical fiber strain sensors [4]. Some of the above techniques yield information that is essentially averaged across the through-thickness direction of a specimen, whilst many of the others reflect surface-specific two-dimensional deformation behavior. Several techniques, including the multiple-embedded-grid moiré method [5], frozen stress photoelasticity [6], the isodyne technique [7], the scattered light speckle photography technique [8] and optical fiber strain sensing [9] enable the assessment of local crack driving forces within a bulk material. However, there are significant limitations with the multiple-embedded-grid moiré method, which only provides interior strains at several points with low sensitivity, whilst the frozen stress photoelasticity, the isodyne and the scattered light speckle photography techniques are limited to transparent and/or birefringent materials. The optical fiber strain sensing approach facilitates measurements between widely separated points (separations of the order of at least several millimeters) making it impossible to examine the effects of microstructural features and local crack geometries. A further fatal drawback of the methods that involve embedded particles or fibers is of course the potential perturbation in the internal stress fields of interest by the incorporation of such dissimilar second phases in a given material.

In practical materials, which usually exhibit complicated fracture behavior, various microscopic fracture events are observed, such as the deflection/twisting of a crack, crack closure during fatigue, microcracking, crack bifurcation, load transfer interactions with secondary phases and crystallographic effects caused by an underlying matrix. These phenomena sometimes shield or anti-shield the crack tip from an applied load, thereby local crack driving forces are assumed to vary 
substantially. However, the abovementioned techniques do not provide a direct estimate of the internal and local crack driving forces at a microstructural level.

To the best of the authors' knowledge, the only conventional method for determining such local and internal cracking information is 3-D numerical simulation. However, previous studies concerned with the simulation of cracking are mostly based on models with significantly simplified geometries such as an idealized microstructure and a flat crack morphology. Such models may not represent the actual local-scale loading conditions of a real crack and thus, may not always clarify the fracture micromechanisms properly. Recently Qian et al. [10, 11] reported a 3-D image-based modeling technique. They visualized a real crack and its propagation process in 3-D by using in-situ high-resolution X-ray computed tomography (CT). The surface planes of a crack and dispersed particles were extracted from tomographic volumes to create an image-based finite element model. They performed a series of numerical simulations considering the real morphologies, spatial distribution and size of the crack and the particles. However, the abovementioned technological limitations also prevent the validation and verification of image-based numerical simulations. Therefore, realizing an advanced procedure for measuring local crack-tip driving forces in 3-D would also clearly be valuable.

The present authors have proposed a 3-D procedure for measuring internal local crack driving forces in a series of high-resolution tomographic images in a companion paper [12]. The physical displacements of microstructural features in a crack-tip stress field are used to calculate local mixed-mode crack driving forces inversely from the displacement field along a crack front line. The proposed technique has provided a highly effective way of assessing local crack driving forces and clarifies, for example, the effects of near-tip contact between crack faces on the effective fatigue crack driving forces. However, the proposed technique does not appear to be versatile. For example, the physical displacement of microstructural features can sometimes be close to the resolution limit (approximately $1 \mu \mathrm{m}$ for conventional projection CT and more than $100 \mathrm{~nm}$ for ultra highresolution imaging CT [13]) even if state-of-the-art synchrotron micro-CT is employed. Also, the 
effects of uncertainty in the location accuracy of a crack tip appear to be non-negligible when the crack-tip opening displacement level is close to voxel size for high strength and low fracture toughness materials. Another factor affecting the accuracy is the unknown transition behavior in crack-tip displacement fields between a damage zone and an elasto-plastic field, and that between the elasto-plastic and elastic fields. Associated errors in the local crack driving force calculation might not be small in certain cases.

To overcome the above shortcomings of the technique proposed in the previous study, this study proposes a more direct measurement of the mode I crack driving force. The 3-D reconstructed volume data of internal features has been employed for the high-density mapping of crack driving forces along a real crack front as a form of local CTOD variation. To confirm the feasibility of this technique, a high-resolution phase contrast imaging technique has been used to obtain crack images and the details of microstructural features in a cast aluminum alloy, which consists of a ductile $\alpha-\mathrm{Al}$ phase and a rather brittle Al-Si eutectic phase [12]. A detailed examination of the 3-D characteristics of the crack-tip region is used to discuss variations in the crack driving force in different phases in terms of the difference in local fracture resistance.

\section{Experimental Methods}

\subsection{Sample preparation}

Al-Si-Mg alloy was prepared using 99.99\% Al, Al-10\% Mg and Al-25.6\%Si master alloys. The alloy melt was kept at $983 \mathrm{~K}$ for $1.8 \mathrm{ks}$, and then poured directly into a steel mould of about $153 \times$ $270 \times 100 \mathrm{~mm}$ in size that had been preheated at $473 \mathrm{~K}$. The melt was modified by the addition of Sr to refine the eutectic Si particles and degassed with Ar gas before casting. The alloy cast had a chemical composition of $6.7 \mathrm{Si}, 0.31 \mathrm{Mg}, 0.12 \mathrm{Fe}, 0.12 \mathrm{Ti}, 0.0035 \mathrm{Sr}$ and balance $\mathrm{Al}$ in mass \%. The samples were then solution heat-treated for $21.6 \mathrm{ks}$ at $813 \mathrm{~K}$, and artificially aged for $21.6 \mathrm{ks}$ at 
$428 \mathrm{~K}$ to provide a T6 temper condition. The alloy consists of a ductile $\alpha$-Al phase and a rather brittle Al-Si eutectic phase, which is fundamentally an aluminum alloy with dispersed spherical Si particles.

Conventional single-edge notched $(\mathrm{SEN})$ specimens $10 \times 10 \times 55 \mathrm{~mm}$ in size were produced with a slit giving a ratio of nominal width, $W$, to an initial notch length $a$ of 0.45 . A fatigue precrack was then introduced into the specimen according to ASTM Standard E 647-95A until $a / W(a$, crack length) became about 0.6 . Small parallelepiped specimens (typically $0.6 \times 0.6 \times 11 \mathrm{~mm}$ ) were carefully machined from the crack-tip region of the fatigue pre-cracked SEN specimens using an electrodischarge machine wire eroder so that $a / W$ for the small parallelepiped specimen became about 0.5. Finally, two end tabs were bonded to each specimen as shown in Fig. 1 to make the sticklike specimen double shouldered for loading in the synchrotron experiments. Note that since the plane strain condition is not satisfied in the present experiment, the plane stress condition is dominant in subsequent in-situ tomography observations.

\subsection{Tomographic imaging}

A high-resolution X-ray CT experiment was performed using the X-ray imaging beamline, BL47$\mathrm{XU}$, of the synchrotron radiation facility, SPring-8, in Hyogo, Japan. A material test rig specially designed for X-ray CT was positioned approximately $49 \mathrm{~m}$ from the X-ray source. A monochromatic X-ray beam with a photon energy of $20 \mathrm{keV}$ produced by a liquid nitrogen cooled Si (111) double crystal monochromator was used for the experiment. A beam diffuser rotating at $600 \mathrm{rpm}$, which was packed with graphite powder, was employed to modify the high coherence of the X-rays. An image detector was positioned $50 \mathrm{~mm}$ behind the sample, thereby making the imaging system sensitive to phase modulation in addition to the classical absorption contrast [14]. The image detector consisted of a cooled $4000(\mathrm{H}) \times 2624(\mathrm{~V})$ element CCD camera (pixel size of the camera: $\left.6.45 \mu \mathrm{m}^{2}\right)$, which was used in a $2 \times 2$ binning mode, a scintillator $\left(\mathrm{Lu}_{2} \mathrm{SiO}_{5}: \mathrm{Ce}\right)$ and a 
relay lens $(\times 20)$. In total 1500 radiographs, scanning 180 degrees, were obtained around the loading axis in 0.12 degree increments for each load level.

The entire cross-section of the specimen and a region about $622 \mu \mathrm{m}$ high containing a crack were captured on the CCD camera. The in-situ loading stage allowed specimens to be scanned under monotonic tensile loading. All the scans were performed while the loaded samples were being held at fixed displacements. The first tomography scan was performed without loading and the subsequent nine scans were performed until crack propagation was observed throughout the width of the specimen as shown in Fig. 2. To suppress the occurrence of blurring caused by the relaxation behavior of the material, the applied displacement was held for $35 \mathrm{~min}$ for aging before each scan, which is seen as a vertical line above a number in Fig. 2. The test rig controller had a load resolution of $0.1 \mathrm{~N}$, which made it possible to undertake well-controlled quasi-static material tests using the miniaturized specimens. The test rig exhibited a displacement stability of about $0.1-0.2$ $\mu \mathrm{m}$, thereby enabling the realization of tomographic scans with almost no blurring caused by specimen wobble.

Image slices were reconstructed from a series of projections based on the conventional filtered backprojection algorithm. The grey value in each dataset was calibrated so that the linear absorption coefficient of $0-30 \mathrm{~cm}^{-1}$ falls within an 8 bit gray scale range between 0 and 255 as shown in Fig. 3 . An isotropic voxel with a $0.474 \mu \mathrm{m}$ edge was achieved in the reconstructed slices.

\section{CTOD Extraction}

As is well known, CTOD is a fracture mechanical parameter that has a unique relationship with the $\mathbf{J}$ integral [15]. It is applicable over the strict limitation in specimen size in the linear elastic fracture mechanics. Unlike the other fracture mechanical parameters, CTOD is a geometric parameter and can be directly measured in a 3-D crack image. This contrasts with the indirect measurement of crack driving forces described in the previous study [12] where the physical 
displacements of marker points are converted to a stress intensity factor or $\mathbf{J}$ integral inversely through the crack-tip displacement field.

The process for determining crack-tip location is shown in Fig. 4. Firstly, a crack-tip voxel is identified on each virtual slice. These slices are parallel to a plane containing mode I crack growth and loading directions. In a previous study, the crack-tip location was assumed to be the center of gravity of the crack-tip voxels that are the farthest from a crack mouth [12]. However, assuming a crack deflecting in the mode II direction, the use of this method might result in the eccentricity of a crack tip in the last deflected crack segment. In this study, an intersection point between the center line of the last deflected crack segment and a crack face is defined as a crack tip. First, the midpoints between the upper and lower crack faces are obtained between horizontal distances of 24 and $95 \mu \mathrm{m}$ from an approximate crack-tip location, thereby extracting the center line of the last deflected crack segment by the linear interpolation of the midpoints. After determining the crack-tip location, a pair of intersecting points of the 90 degree vertex taken from a crack tip with crack flanks is determined to measure the CTOD value in the local crack-tip coordinate system with the center line of the last crack segment as a horizontal axis. When there are uncracked ligaments between the last deflected crack segment and an actual crack tip, the most advanced point in the crack-tip coordinate is considered to be a crack-tip location. The average crack mouth opening displacement (CMOD) is also measured in tomographic images to estimate the CTOD value with the conventional plastic hinge model [16].

Actual crack extension is accompanied by various complex phenomena, such as microcracking, bifurcation, and uncracked ligaments, depending on the effects of the underlying microstructure. A careful slice-by-slice check was made in a preliminary investigation to ascertain whether the cracktip location is determined with the above method. Figure 5 shows three typical slices where cracktip voxels are located in (a) the $\alpha$-Al phase, (b) the eutectic Al-Si phase and (c) an interfacial region between the two phases. In the $\alpha$-Al phase, crack-tip blunting occurs stably to a larger extent than in the eutectic Al-Si phase as shown in Fig. 5 (a). There is no difficulty in obtaining CTOD in such a 
case. In Fig. 5 (b), small isolated crack segments are observed ahead of the main crack. However, this is not microcracking or an uncracked ligament but premature cracking in the eutectic Al-Si phase, which is in fact connected to the main crack on some underlying slices. This has been determined with a three-dimensional 'seed' growth technique by identifying the volume of the crack. In the procedure, all voxels that are three-dimensionally connected to the crack and that have a voxel grey value within a set tolerance range are segmented and labeled as one main crack. The CTOD value for Fig. 5 (b) was measured within the apparently isolated crack segment, resulting in a relatively small CTOD value. In Fig. 5 (c), where the crack tip is propagating from the $\alpha$-Al phase to the Al-Si eutectic phase, a complex crack-tip configuration is observed. It has been confirmed that the above method works well even in such a case.

\section{Fracture Behavior}

Figure 6 shows the 3-D distribution of eutectic silicon particles within part of a rendered volume $(525 \times 474 \times 273 \mu \mathrm{m})$ encompassing the front line of a crack. The images were captured at four different loading steps specified in Fig. 2. A large number of particles (shown in red) are observed in the volumes. Almost no damage initiation was observed before the fifth loading step. Ductile crack blunting was observed in subsequent loading steps, which was accompanied by damage evolution at the silicon particles. Voids, which are a typical feature of the ductile fracture process, are shown in green in Fig. 6. There are two possible formation mechanisms for these voids. One is the general cavity formation associated with broken brittle particles that fail during loading [17]. A second void formation mechanism has been reported recently $[18,19]$, namely the homogeneous or heterogeneous precipitation of hydrogen at a high temperature mainly during heat treatment prior to loading. Such pre-existing micro-pores are also-asily observed in the microstructure of the alloy used, and they are dissociated from the silicon particles. The overall poresity was measured at about 
eavity associated with damage is about $0.7 \%$. As is well known, in a ductile material such as the Al-Si alloy used here, there is a large strain region, where the small strain theory breaks down because the strains exceed $10 \%$. The size of the large strain region corresponds to approximately twice the CTOD value. Damage evolution is observed extensively ahead of the crack tip as shown in Fig. 6 (b) and (c), and appears to extend far beyond the large strain region. During the last few steps, stable crack extension is observed from the tip of the fatigue pre-crack as shown in Fig. 6 (d). The crack-tip configuration appears to be sharp, which is typical of stable crack extension in a ductile material. The mechanism of the transition in the crack-tip configuration is understood to relate to the fact that the material behind a growing crack unloads elastically.

In Fig. 7, only crack images were extracted from the tomographic volume and they are shown as 3-D perspective images viewed in the mode I loading direction. The crack images, which are substantially the air gap between the upper and lower crack faces, have been segmented and extracted by means of the 3-D seed growth technique described above. The underlying aluminum and other microstructural features such as micro-pores, intermetallic compound particles and silicon particles are not displayed here. The complicated and irregular patterns observed in each image are fracture surface roughness. These are rendered by using front and side diffuse light sources to highlight the three-dimensional nature of the crack. The crack extension varied greatly with the $x$ coordinate (i.e. along the crack front line) within the current tomography specimen as also observed previously in 3-D images $[12,20]$. The retarded section of the crack front is seen around the center of the crack front line (i.e. a slice number between about 250 and 500), which is especially obvious in Fig. 7 (c). On the other hand, a localized crack advance with a width of $10 \sim 50 \mu \mathrm{m}$ can be clearly observed at slice numbers above 400 in Fig. 7 (b). Such a marked difference between the two regions might be attributed to the difference in local fracture resistance. In fact, the underlying phases have been identified as ductile $\alpha$-Al phase and the rather brittle Al-Si eutectic phase for the retarded and locally advanced crack segments, respectively, by investigating the original 
tomographic volumes. Typical examples are shown by the virtual cross-sections in Fig. 8 (a) and (d), which correspond to slice numbers 312 and 527, respectively. The crack tip is located at the center of an $\alpha$-aluminum dendrite with an irregular shape, which is typical of the material in Fig. 8 (a), while in Fig. 8 (d) the crack tip is adjacent to the even more irregular eutectic phase. Gradual crack extension and extensive damage distribution are also identified in a series of virtual cross-sections in Fig. 8 (d) - (f) in which the crack tip is propagated through the eutectic phase.

Another noteworthy feature is the subsequent crack propagation behavior. Between Fig. 7 (c) and (d), there appears to be crack extension along the whole crack front line. However, a closer inspection has revealed that the prematurely initiated crack segments ahead of the aluminum-silicon eutectic phase, which are seen between slice numbers of about 500 and 800 in Fig. 7 (b), were then further extended in the lateral direction. Therefore the crack extension in the $\alpha$-Al phase observed at slice numbers between about 250 and 500 in Fig. 7 (c) is not crack re-initiation from the blunted crack tip, but simply a lateral crack extension from the neighboring Al-Si eutectic phase. The mode I crack driving force near the blunted crack segment might arise owing to the existence of the neighboring advanced crack segments, while that at the locally advanced crack segments is effectively decreased as a result of the constraint imposed by surrounding uncracked sections. Therefore it can be inferred that the local crack advance in the Al-Si eutectic phase is interrupted at some stage, and the lateral extension from the eutectic phase might be promoted ahead of the $\alpha-\mathrm{Al}$ phase.

In Fig. 7 (d), more than ten white patches remain in the newly extended crack segment. These are referred to as uncracked ligaments in traditional cross-sectional observations. Its mechanism and the subsequent crack bridging are well documented in the literature [21]. It can be confirmed from the virtual cross-section shown in Fig. 8 (c) that the new crack tip is located in the eutectic phase ahead of the former crack tip, and the $\alpha$-Al phase regions are left intact in between. A similar uncracked ligament has been reported in a post-overloaded fatigue crack extension [12]. 
The observation of the virtual cross-section in Fig. 8 (a) - (c) also reveals multiple crack planes overlapping in the $x$ direction. The shorter overlapped crack plane exhibits significant local mode II crack deflection (i.e. crack deviation about the $y$ axis parallel to the macroscopic crack growth direction). This gradually disappears on neighboring $y z$ slices if gone to the $+x$ direction, while the major crack segment disappears if gone to the $-x$ direction. This sort of 3 -D crack bifurcation has been frequently observed in close inspections of tomography data $[12,22,23]$, revealing that such complex arrangements of crack planes probably interact intricately with the underlying crystallographic grain structure. In a previous study, a combined methodology consisting of in-situ 3-D crack visualization and image-based crack simulation has demonstrated that one crack segment significantly affects the fracture behavior of another neighboring hidden or only partly overlapped crack segment by shielding a near-tip stress/strain field and shifting the near-tip plastic zone of a crack [23]. In such cases, we can assume the existence of a grain boundary between the two crack segments. The previous study showed that the magnitude of a near-tip stress/strain field is effectively reduced, thus leading to the retarded propagation of a crack front near an overlapped region [23]. Therefore high local fracture resistance is expected around the crack bifurcation seen in Fig. 8 (a) - (c), which will be proved quantitatively later in Fig. 10 according to the procedure proposed here.

\section{Crack driving force variation}

\subsection{Feasibility of tracking Si particles to measure crack driving force in present material}

To examine the applicability of the previously reported 3-D measurement procedure for internal local crack driving forces, which is based on a crack-tip displacement field [12], the centroid location for each silicon particle was tracked against a corresponding crack tip as a function of applied load. Compared with the original procedure [12], accuracy and reliability have recently 
been significantly improved by employing sophisticated tracking algorithms [24]. The tracking method consists of the application of a matching probability parameter, a macroscopic trajectory prediction and a spring model tracking algorithm, which is applied where the clustering of markers makes accurate tracking impossible. By combining these three algorithms, it has been demonstrated that tracking error can be minimized to an almost negligible level in practical materials $[24,25]$. It is crucial to realize automatic high-density mapping in practical materials, which inherently contain small and closely interspersed microstructural features. It has also been clarified that the tracking results are relatively independent of the parameter setting conditions and such stability is beneficial from a practical point of view.

Figure 9 shows an example of an obtained displacement field displayed on a virtual crosssection in the material used. Since displacement vectors are apparently random, the data seem not to provide any valuable information on local crack driving forces. It can be inferred that such tracking errors are directly associated with a significant agglomeration of the silicon particles of similar size and shape in the present material.

\subsection{Measured CTOD and its interpretation}

Figure 10 shows measured CTOD variations along the crack front line, which are superposed on a set of the same front and side illuminated renderings of the crack images as those in Fig. 7. The CTOD value is relatively large where the crack front line is located in the ductile $\alpha$-Al phase (i.e. slice number less than 415). At loading step 3 in Fig. 10 (a), where an almost linear loaddisplacement relationship is still maintained in Fig. 2, the measured CTOD values are overly small even for crack-tip locations in the ductile $\alpha$-Al phase, indicating that the crack-tip deformation is confined mostly to elastic deformation. At loading step 6, the CTOD level increases below a slice number of about 450 , and considerable scattering in the CTOD is also observed. This scattering is partly because crack bifurcation is observed between slice numbers 200 and 500, and the obliquely 
propagating minor crack segment becomes longer as the slice number decreases. Another reason for the scattering is the complex spatial distribution of the $\alpha$-Al and Al-Si eutectic phases around the crack tip. It should be noted that CTOD level is even more scattered for slice numbers below 250. In this region, the crack front line is located in the vicinity of an $\alpha$-Al/eutectic phase interface. Therefore some early crack extension is observed as seen in the eutectic phase (i.e. slice number above 500). At the last loading step, however, all of the measuring points exhibit small CTOD values. This is because almost all the crack-tip segments are located in the Al-Si eutectic phase owing to the characteristic crack growth behavior mentioned above.

Measured CTOD variations with crack advance are shown in Fig. 11 superposed on corresponding virtual cross-sections. Crack-tip locations and crack paths are also shown in the figure. In Fig. 11 (a) - (b), the initial crack tip is located in the $\alpha$-Al phase, while in Fig. 11 (c) and (d) it is located in the eutectic phase. In Fig. 11 (c), the crack tip is located in the vicinity of the $\alpha$ $\mathrm{Al} /$ eutectic phase interface. The apparent crack extensions observed during the initial $1-2$ steps are actually caused by the fatigue crack closure behavior. The CTOD value increases with loading in (a) - (b), and then decreases immediately after a crack extension is observed. The CTOD value appears to be dependent on local ductility around the crack tip (i.e. the local fraction of the $\alpha$-Al phase in the vicinity of the crack tip). After the onset of crack propagation, the crack-tip location jumped over $50-100 \mu \mathrm{m}$ leaving uncracked ligaments in the $\alpha$-Al phase. For the crack extension in the eutectic phase, gradual crack extension is observed as typically shown in Fig. 11 (d) and the CTOD value remains between 0 and $5 \mu \mathrm{m}$.

Typical changes in the CTOD with applied displacement are shown in Fig. 12 for eight different slices (four slices with crack-tip locations in the $\alpha$-Al phase in Fig. 12 (a) and four with crack-tip locations in the eutectic phase in Fig. 12 (b)). A significant drop in the CTOD is observed between the 9th and 10th loading steps in Fig. 12 (a), indicating the onset of crack extension between these loading steps in the $\alpha$-Al phase. The maximum CTOD value immediately before the crack extension increases with increases in slice number. This is probably because of the effects of the 
crack bifurcation described above. Similar decreases in the CTOD after crack extension are also observed in Fig. 12 (b), indicating that the onset of crack extension occurs at the 6th or 7th loading step in the eutectic phase.

Figure 13 shows the average CTOD values for each phase as well as the overall averages and CTOD values estimated from the average CMOD obtained with the conventional plastic hinge model. The CTOD value for each phase gradually increases with external loading and exhibits its maximum values at the 7th and 9th loading steps for the eutectic and $\alpha-\mathrm{Al}$ phases, respectively. The two peak CTOD values represent the critical CTOD values that reflect the local fracture toughness of the two phases, suggesting that the local fracture toughness value of the $\alpha$-Al phase is about six times larger than that of the eutectic phase. Although the volume fractions of the $\alpha$-Al and eutectic phases are almost the same, the total fracture resistance of the whole material expressed by $\delta_{0}$ is slightly smaller than the intermediate value between the two values. This is attributable to the abovementioned preferential crack growth through the eutectic phase.

It is also noted that the estimation of the CTOD values from the macroscopic quantity (i.e. CMOD) significantly overestimates the magnitude of the fracture toughness. This is probably because of the unrealistic assumption in the plastic hinge model that crack faces are rigid and plastic deformation occurs within a limited narrow strip with a virtual point as an imaginary rotational center. The CTOD converted from the CMOD increases monotonically even after the onset of crack propagation and the folding point is so subtle that it might go unnoticed. It can be concluded that the conventional CTOD criterion based on the CMOD measurement does not reflect crack-tip deformation in a reasonable way, which is in contrast to the direct measurement procedure proposed in the present study.

It is interesting to note that transition behavior in the CTOD is observed across the $\alpha$-Al phase/eutectic phase interface as shown in Fig. 14. The transition region is shifted right and left from its original location with loading because the interface is geometrically complex in 3-D. The transition is observed over about 10 and $15 \mu \mathrm{m}$ at the 7 th and 9th loading steps, respectively. This 
implies that a scaling factor is introduced into the fracture/microstructure relationship in dual phase materials such as the present material. If the size of each phase is properly controlled so that it is as small as the transition region during its production process, it can be expected that premature crack extension in a more brittle phase will be suppressed and the overall fracture toughness improved.

\subsection{Comparison with displacement field based crack driving force measurement procedure}

Advantages, disadvantages and differences as regards the two local crack driving force measurement procedures are discussed below. In the displacement field based method, which is reported in a companion paper [12], a crack driving force value calculated from the physical displacement of each microstructural feature, which is in general a few tens to a few hundreds of micrometers from a crack tip, is allocated to the nearest crack-tip location. It is assumed that the effect of the deformation and damage behavior of neighboring regions operates in the direction of the central axis in a cylindrical coordinate system that has a crack front line as its central axis .The effect of the accumulation of deformation/damage between the crack tip and the microstructural feature operates in the radial direction of the cylindrical coordinate system. It can readily be said that the displacement field based method would provide average information over a relatively wide region rather than the fracture resistance at the point where a crack tip is presently located. The azimuth angle from a crack tip to each microstructural feature would also have some effect in terms of the difference in underlying texture and damage, giving rise to scattering in measured CTOD values depending on the location of the strain marker point in relation to the crack tip. On the other hand, with the present direct measurement method it can be expected that the local fracture resistance at which the crack tip is located can be directly measured without failure or reproducibility error. It can be concluded that the present method would be particularly effective for

revealing the microstructure/fracture relationship in complex 3-D materials. It can also be expected that the present method will be more sensitive to near-tip fatigue crack closure behavior, local crack 
advance, local crack configuration such as deflection and bifurcation, and local damage than the displacement field based method.

With the displacement field based method, microstructural features such as particles and pores, which are appropriately larger than a voxel size, should be homogeneously dispersed in a high density. Generally, a sufficient number of such microstructural features are available in most industrial materials. However, the driving force cannot be measured when particles or pores of similar size and shape are agglomerated as shown in Fig. 9. In addition, when microstructural features are not sufficiently small compared with the crack-tip stress field, the measurement becomes less accurate, because the existence of the microstructural features affects the displacement field. In dual phase materials such as the present material and ferrite/pearlite steel, accurate measurement is difficult owing to the distortion of the crack-tip displacement field. Another problem to note is that the 3-D crack-tip displacement field cannot be verified due to the lack of other experimental measuring methods. In contrast, there is a well-established conventional method for measuring the CTOD on a fracture surface as the stretch zone width after fracture. CTOD measurement from 3-D images can therefore be readily verified by obtaining critical CTOD values on a fracture surface for ductile materials with uniform microstructures.

Light and dark fringes at each crack edge induced by the phase contrast effect cause location measurement errors that at their maximum exceed half the voxel size (i.e. about $0.24 \mu \mathrm{m}$ ) in this study. Moreover, the so-called partial volume effect [26], which induces a transition area with a grey value ramp over several voxels in width at the interface between radiographically dissimilar phases, may also be crucial. This is in principle attributed to the degradation in resolution for various reasons. This effect can also cause a maximum error of half the voxel size when determining the crack-tip location. In the previously reported procedure based on the displacement field, near-tip particles or pores are used as strain markers [12]. The distance between a crack tip and the markers can be as small as a few times the minimum CTOD level, which is less than $5 \mu \mathrm{m}$ in the eutectic phase of the present alloy. This corresponds to around $10-20$ times the errors. As 
such, the average errors due to such uncertainty in the location accuracy of a crack tip are not necessarily negligible for the displacement field based driving force calculation. However, when the CTOD is measured, variations caused by such uncertainty in the location accuracy of the crack tip appear to be small because the curvature of the crack face is not so large at the point where the CTOD is measured.

It should be noted that the effects of minor mode III displacement on fatigue crack closure behavior are clarified in the previous work [12]. The displacement field based method can therefore be used to measure small mode II and/or mode III driving forces and employed in cases where mode II and/or mode III displacement is predominant. On the other hand, with the present method, it might be difficult to track a feature on a crack face in the mode II and III directions without failure. Accuracy might also be reduced if significant crack deflection or tilting occurs. It can therefore be considered that the present method is suitable for fracture resistance evaluation when mode I loading is predominant. The physical displacement of a microstructural feature increases with the distance from a crack tip, and in general is greater than the CTOD value. When the cracktip plasticity is limited and the CTOD value is not larger than several times the voxel size, there is no alternative but to employ the displacement field based method.

\section{The variations in crack driving force before and after crack extension, which have only} previously been measured on a specimen surface, have been revealed for the first time inside the material with the present method. The developed technique might provide ways of assessing a growing crack and confirming the theory of growing cracks for the first time. For example, Chan proposed a criterion for stable crack growth on the basis of the mechanies of a growing crack [27], concluding that stable crack extension is realized as a result of sufficient plastic deformation when the following $\Omega$ exceeds 34.5 , while a crack is propagated without experiencing any propagation resistance when $\Omega$ is less than 34.5;

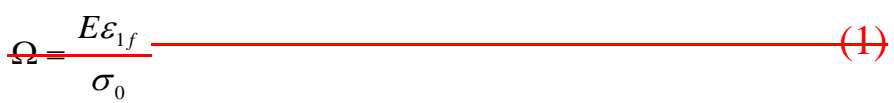


where $\varepsilon_{1 f}$ is the uni axial strain at a fracture, $E$ is Young's modulus and $\sigma_{\theta}$ is flow stress. A simple question might arise as to whether a distinct transition can occur at a location that is determined with a combination of a limited number of uni-axial parameters. The present method might offer the unique possibility of verifying such a phenomenon experimentally for opaque materials.

\section{Summary}

High-resolution synchrotron X-ray microtomography has been used to estimate local crack driving forces at arbitrary crack-tip locations as a form of CTOD. To demonstrate this, the in-situ crack opening and propagation behavior in a cast aluminum alloy was observed at a synchrotron radiation facility. The high-resolution experimental setup and the utilization of phase contrast imaging have made it possible to reconstruct distinct 2D slices and 3D renderings of a crack and underlying microstructural features. Local CTOD variations with external loading have been successfully obtained using the proposed procedure, providing several valuable insights regarding our understanding of crack initiation and propagation behavior through a dual phase material with a complex microstructure. For example, sharp drops in local CTOD have been observed after the onset of crack extension. The procedure has been used to assess the local crack advance. The local fracture resistance in the two constituent phases has also been obtained owing to this variation, and then utilized to explain characteristic crack growth behavior through the Al-Si alloy used. The absolute level of the measured CTOD values was very small compared with values obtained using conventional macroscopic measurement, suggesting that there is an essential defect in the simplified conventional model for CTOD measurement. The existence of the interesting transition behavior in the CTOD across an interface between the two phases has also been confirmed. This may have the potential to be applied to the microstructural design of high fracture resistance dual phase materials.

Overall, the method proposed in the present study is considered to be a robust procedure that is appropriate for automatic high-density mapping with rather ductile practical materials. The 
advantages and disadvantages of the present method have been discussed compared with the displacement field based method developed in the companion paper.

\section{Acknowledgements}

The synchrotron radiation experiments were performed with the approval of JASRI through proposal number 2005A0417-ND1c-np and 2007B1080. This work was partly undertaken as part of the Grant-in-aid for Scientific Research from JSPS through subject Nos. 17360340 and 16•04370. The support of the Tatematsu Foundation and the Light Metal Educational Foundation is also gratefully acknowledged.

\section{References}

[1] Ross EL, Kamiski G, Conway JC. Expl Mech 1982; 22: 117.

[2] Post D. Handbook on Experimental Mechanics. In: Kobayashi AS, editor. Bethel (CT): Society for Experimental Mechanics, 1986. Chap. 7

[3] Davidson DL, Hudak SJ. Metall Mater Trans A 1995; 26: 2247.

[4] Narendran N, Shukla A, Letcher S. Engng Fract Mech 1991; 38: 491.

[5] Xiao QZ, Karihaloo BL. Engng Fract Mech 2001; 69: 959.

[6] Smith DG, Smith CW. Engng Fract Mech 1972; 4: 357.

[7] Pindea JT, Krasnowski BR. Fracture Problems and Solutions in the Energy Industry. In: Simpson LA, editor. New York (NY):Pergamon Press, 1982. p.147.

[8] Chiang FP, Asundi A. Optical Engineering 1982; 21: 570.

[9] Gran FJ, Gysler A, Lutjering G. Fatigue '93. In: Bailon JP, Dickson JI, editors. Warrington: E M A S Ltd., 1993. p.623.

[10] Qian L, Toda H, Nishido S, Akahori T, Niinomi M, Kobayashi T, Metall Mater Trans A 2005;36A,:2979-2992.

[11]Qian L, Toda H, Nishido S, Kobayashi T, Acta Mater 2006;54:4881.

[12] Toda H, Sinclair I, Buffière J-Y, Maire E, Khor KH, Gregson P, Kobayashi T, Acta Mater 2004;52:1305.

[13] Toda H, Uesugi K, Takeuchi A, Minami K, Kobayashi M, Kobayashi T. Appl Phys Lett 2006;89:143112

[14] Cloetens P, Pateyron-Salome M, Buffière JY, et al. J Appl Phys 1997;81:5878.

[15] Anderson TL, Fracture Mechanics, second edition. Boca Raton (FL): CRC Press, 1995. p.117.

[16] Annual book of ASTM Standards. E 1290-93, Standard test method for crack-tip opening displacement (CTOD) fracture toughness measurement, Philadelphia (PA):ASTM,1996.

[17] Zhang H, Toda H, Hara H, Kobayashi M, Kobayashi T, Sugiyama D, Kuroda N. Metall Mater Trans A 2007;38A:1774.

[18] Toda H, Hidaka T, Kobayashi M, Kobayashi T, Uesugi K, Suzuki Y. Proc 10th Asian Foundry Congress $2008 ;: 121$.

[19]Toda H, Hidaka T, Minami K, Kobayashi M, Uesugi K, Suzuki Y, Kobayashi T. Proc ICAA-11 2008;in press.

[20] Qian L, Toda H, Uesugi K, Kobayashi T, Ohgaki T, Kobayashi M. Appl Phys Lett 2005;87:241907.

[21] Shang, J.H. and Ritchie, R.O., Metall. Trans. A, Vol.20A (1989), pp.897-908.

[22] Toda H, Sinclair I, Buffière JY, Maire E, Connolley T, Joyce M, Khor KH, Gregson P. Phil Mag A 2003;83:2429.

[23] Qian L, Toda H, Uesugi K, Kobayashi M, Kobayashi T. Phys Rev Lett 2008;100:115505.

[24]Kobayashi M, Toda H, Kawai Y, Ohgaki T, Uesugi K, Wilkinson DS, Kobayashi T, Aoki Y, Nakazawa M. 
Acta Mater 2008;56:2167.

[25] Miyawaki A, Toda H, Kobayashi M, Qian L, Kobayashi T, Uesugi K. unpublished work, to be submitted to Acta Mater.

[26] Labbe C, Froment JC, Kennedy A, Ashburner J, Cinotti L. Alzheimer Disease \& Associated Disorders 1996; $10: 141$.

[27]Chan KS, Acta Metall 1989;37:1217.

Fig.1 A miniature bar machined from the three-point bending specimen is shown in (a). A couple of end tabs were bonded to prepare a miniature tomographic specimen shown in (b) for the in-situ fracture test. The scale on the ruler is in millimeters.

Fig.2 Load-displacement curve during the in-situ observation of crack initiation and propagation together with crack-mouth opening displacement with loading.

Fig.3 16bit linear absorption coefficient (LAC) value distribution in the reconstructed 3D volume, showing the peaks of air and aluminum. 8bit gray value distribution between 0 and 255 has been extracted from 0 to 30 in the LAC value.

Fig.4 Schematic illustration of the CTOD measurement method together with the global and local coordinates. Fig.5 Typical crack configurations on several tomographic slices with measured deflection angles, crack tip locations and measured CTOD values.

Fig.6 3D perspective views of the loading process. Crack opening, its blunting, damage initiation around a cracktip and the subsequent onset of crack propagation are clearly visualized together with distinct microstructural image.

Fig.7 3D perspective views of crack propagation process viewed from the $z$ direction specified in Fig. 6 .

Fig. 8 Tomographic slices showing the crack initiation and propagation in ductile a-Al phase in (a) - (c) and a rather brittle Al-Si eutectic phase in (d) - (f), which correspond to slice numbers 312 and 527 (Specified in Fig. 7), respectively. Note that in (c) a void-like region is seen ahead of the crack-tip, which is actually connected to the main crack.

Fig.9 Result of the particle tracking indicating the amount and direction of particle displacement for each particle by a white arrow.

Fig.10 Comparison of the top views of the crack and the distributions of CTOD among different loading steps. Fig.11 Tomographic slices showing the variations in crack tip location with crack blunting and propagation on four different slices together with CTOD variations. Note that the crack tip was initially located within an a-Al phase in (a) and (b), in which uncracked lighaments were left after the final crack extension, while in (c) and (d) the crack tip was mainly tracing through an Al-Si eutectic phase and more gradual and continuous crack extension was observed.

Fig. 12 Variations of CTOD as a function of applied displacement for different slices. (a) shows four examples for initial crack tip location in an a-Al phase, while (b) shows those for initial crack tip location in an Al-Si eutectic phase.

Fig. 13 Comparison of the CTOD values obtained by the present $(d 0)$ and conventional macroscopic $(d \mathrm{CMOD})$ methods. $d$ CMOD is calculated based on a single CMOD value with assuming a virtual rotational center, while $d \mathrm{a}$, $d \mathrm{EU}$ and $d 0$ are the measured values averaged over the $\mathrm{a}-\mathrm{Al}$ and the $\mathrm{Al}-\mathrm{Si}$ eutectic phases and the whole crack, respectively.

Fig. 14 CTOD variations across an interface between a-Al and Al-Si eutectic phases at five different loading steps (i.e. the amount of crack extension). Obvious transitions can be identified for the 6th, 7th, 8th and 9th loading steps. The transition occurs over $15 \mathrm{~mm}$ approximately at a maximum. CTOD means the averaged value over the whole crack. 


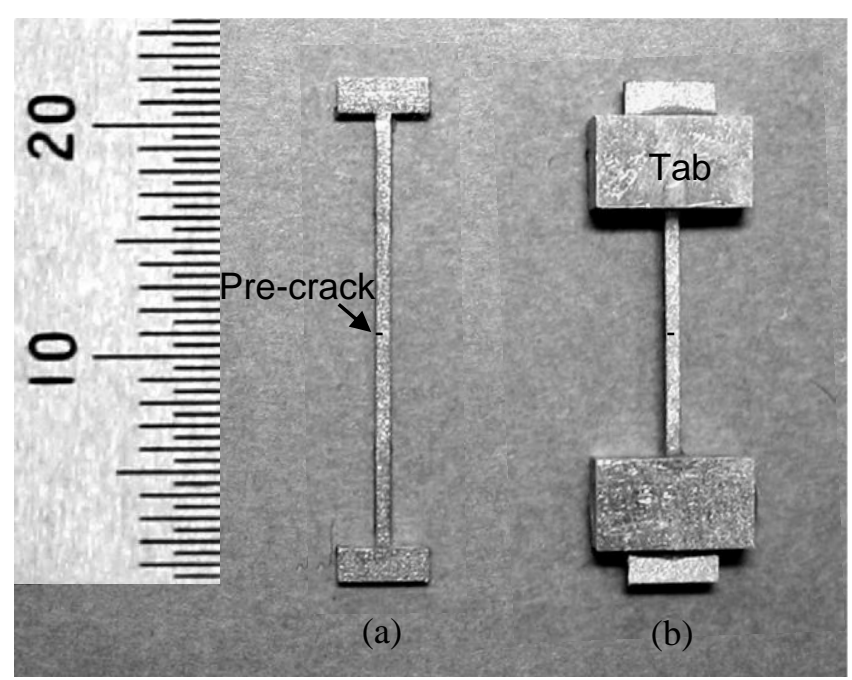

Fig.1 A miniature bar machined from the three-point bending specimen is shown in (a). A couple of end tabs were bonded to prepare a miniature tomographic specimen shown in (b) for the in-situ fracture test. The scale on the ruler is in millimeters. 


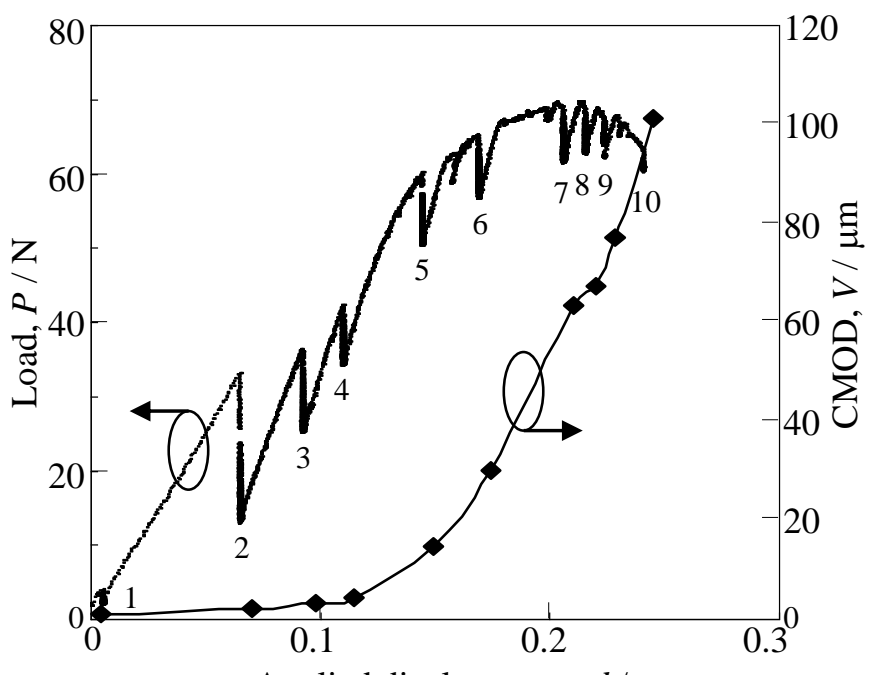

Applied displacement, $d / \mathrm{mm}$

Fig.2 Load-displacement curve during the in-situ observation of crack initiation and propagation together with crack-mouth opening displacement with loading. 


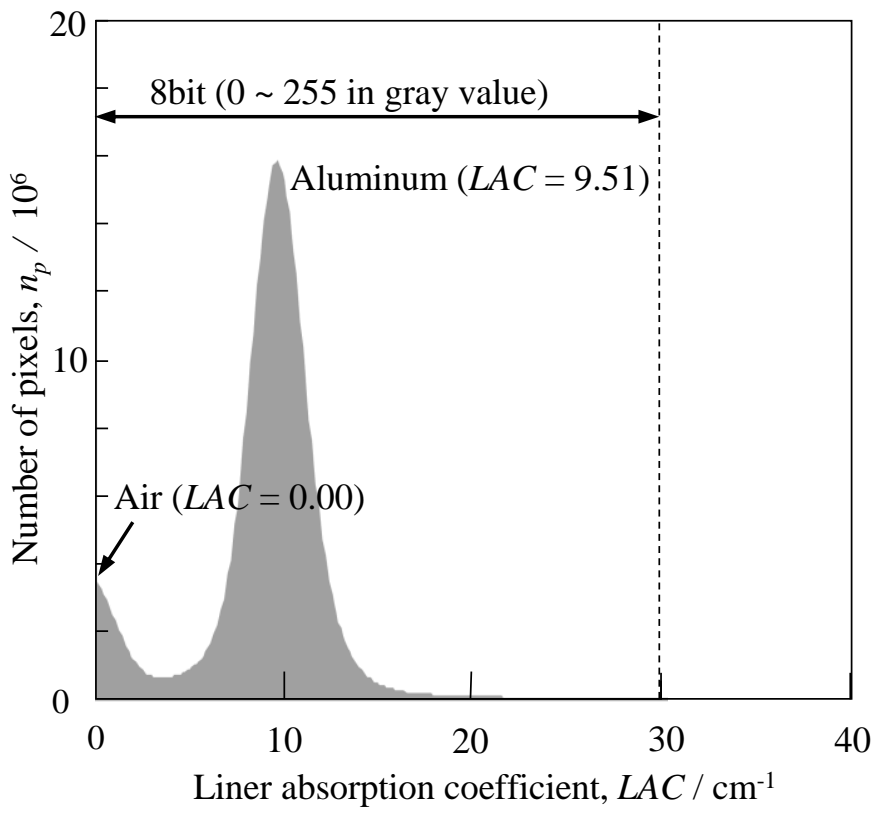

Fig.3 16bit linear absorption coefficient (LAC) value distribution in the reconstructed $3 \mathrm{D}$ volume, showing the peaks of air and aluminum. 8bit gray value distribution between 0 and 255 has been extracted from 0 to 30 in the LAC value. 


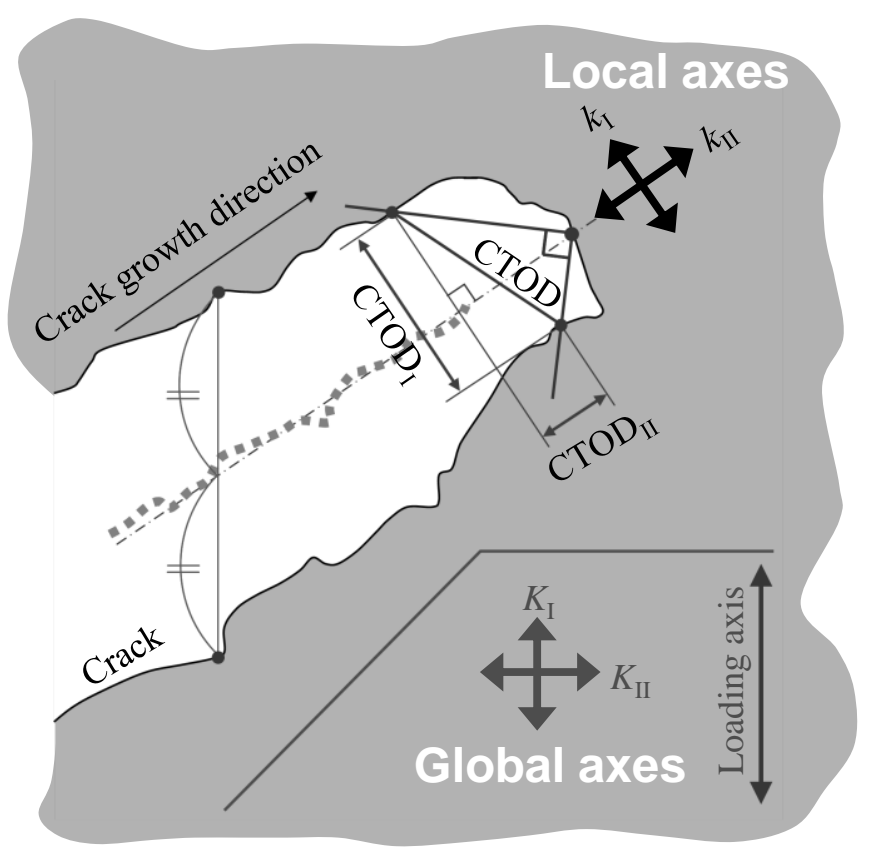

Fig.4 Schematic illustration of the CTOD measurement method together with the global and local coordinates. 


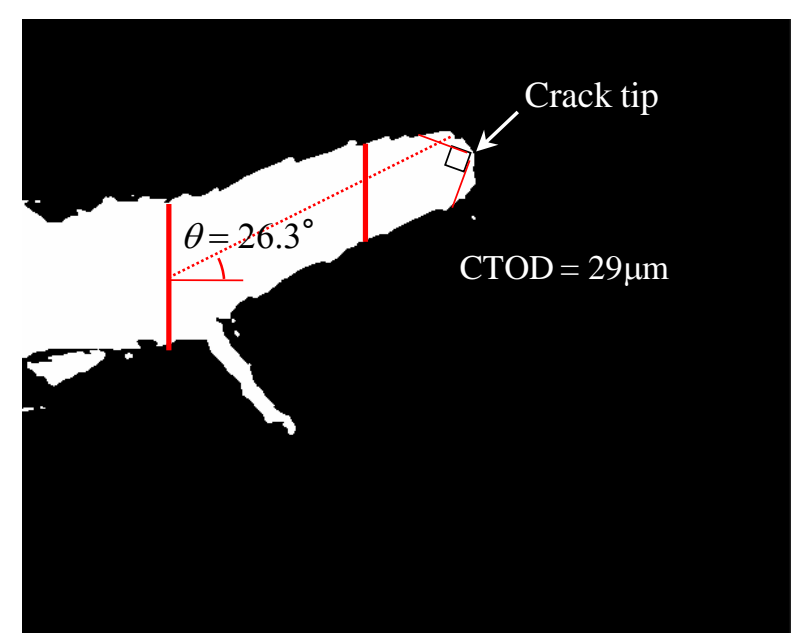

(a) Slice 401

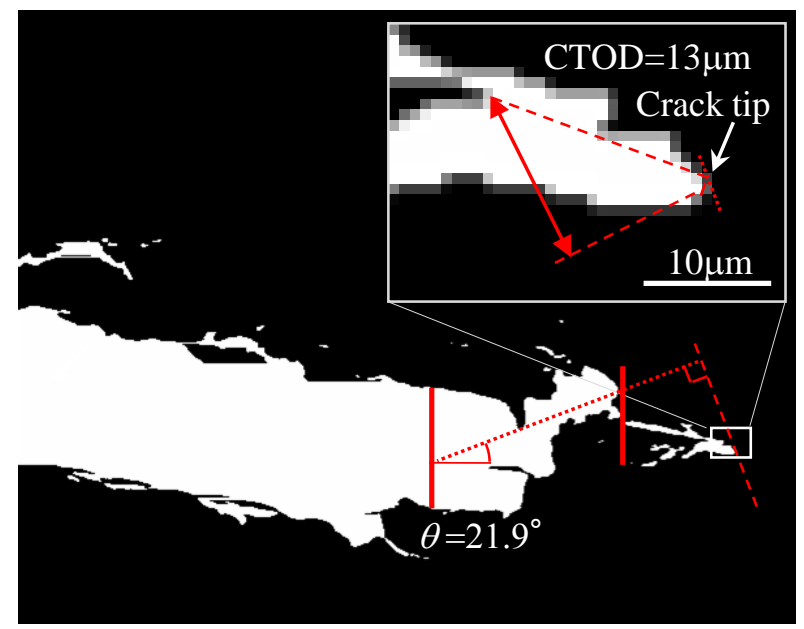

(b) Slice 679

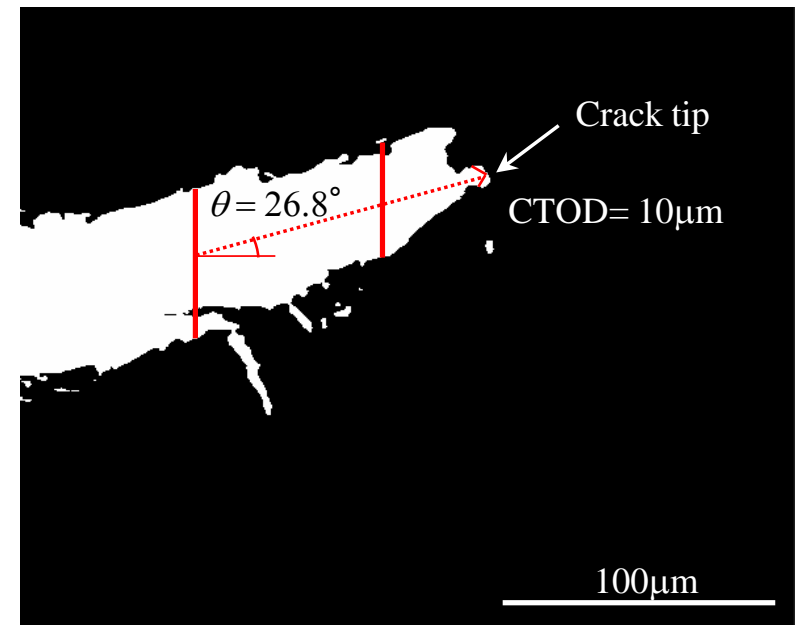

(c) Slice 370

Fig.5 Typical crack configurations on several tomographic slices with measured deflection angles, crack tip locations and measured CTOD values. 


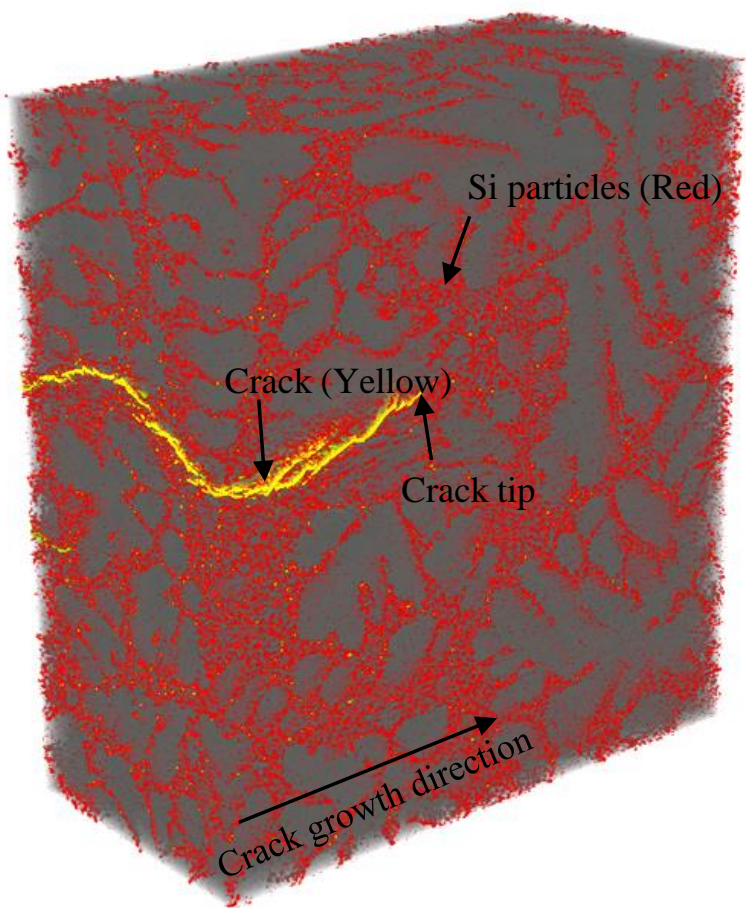

(a) 3rd loading step

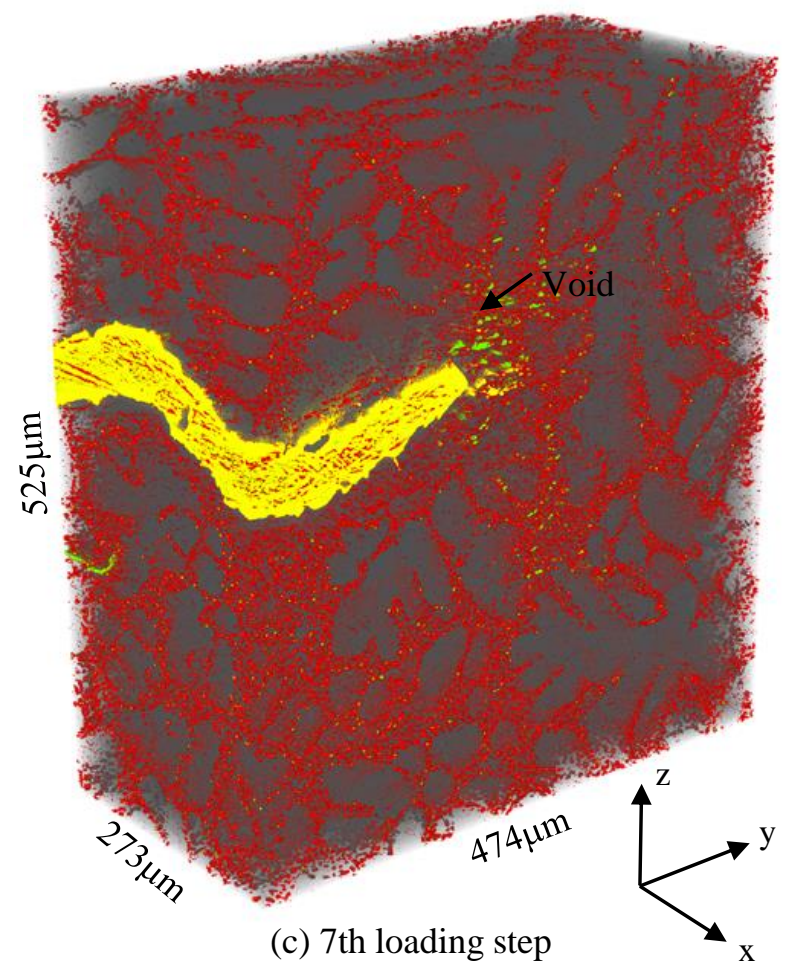

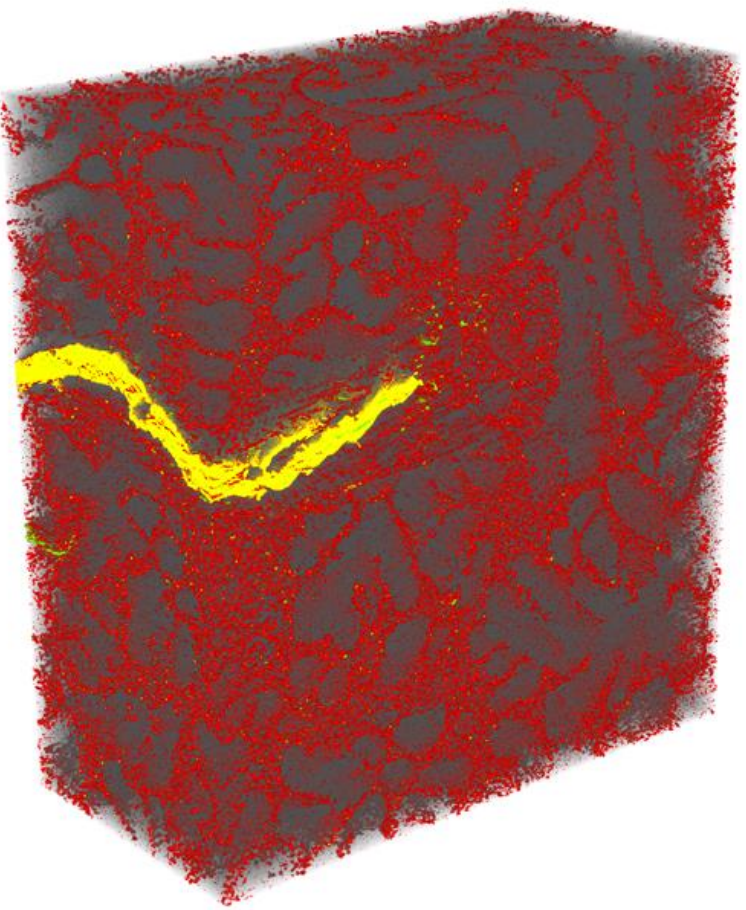

(b) 6th loading step

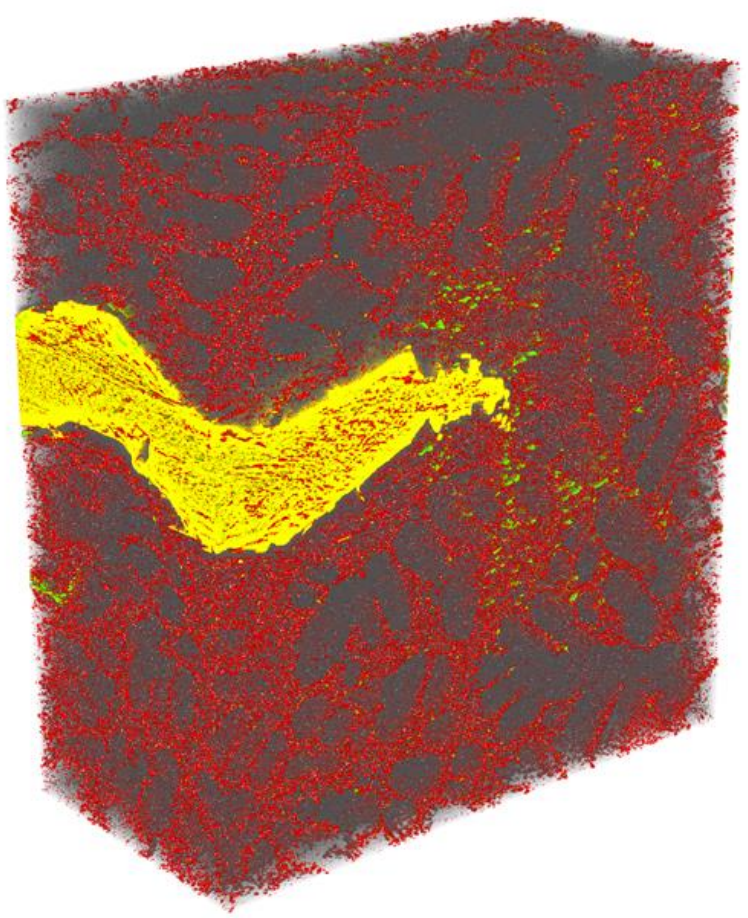

(d) 10th loading step

Fig.6 3D perspective views of the loading process. Crack opening, its blunting, damage initiation around a crack-tip and the subsequent onset of crack propagation are clearly visualized together with distinct microstructural image. 

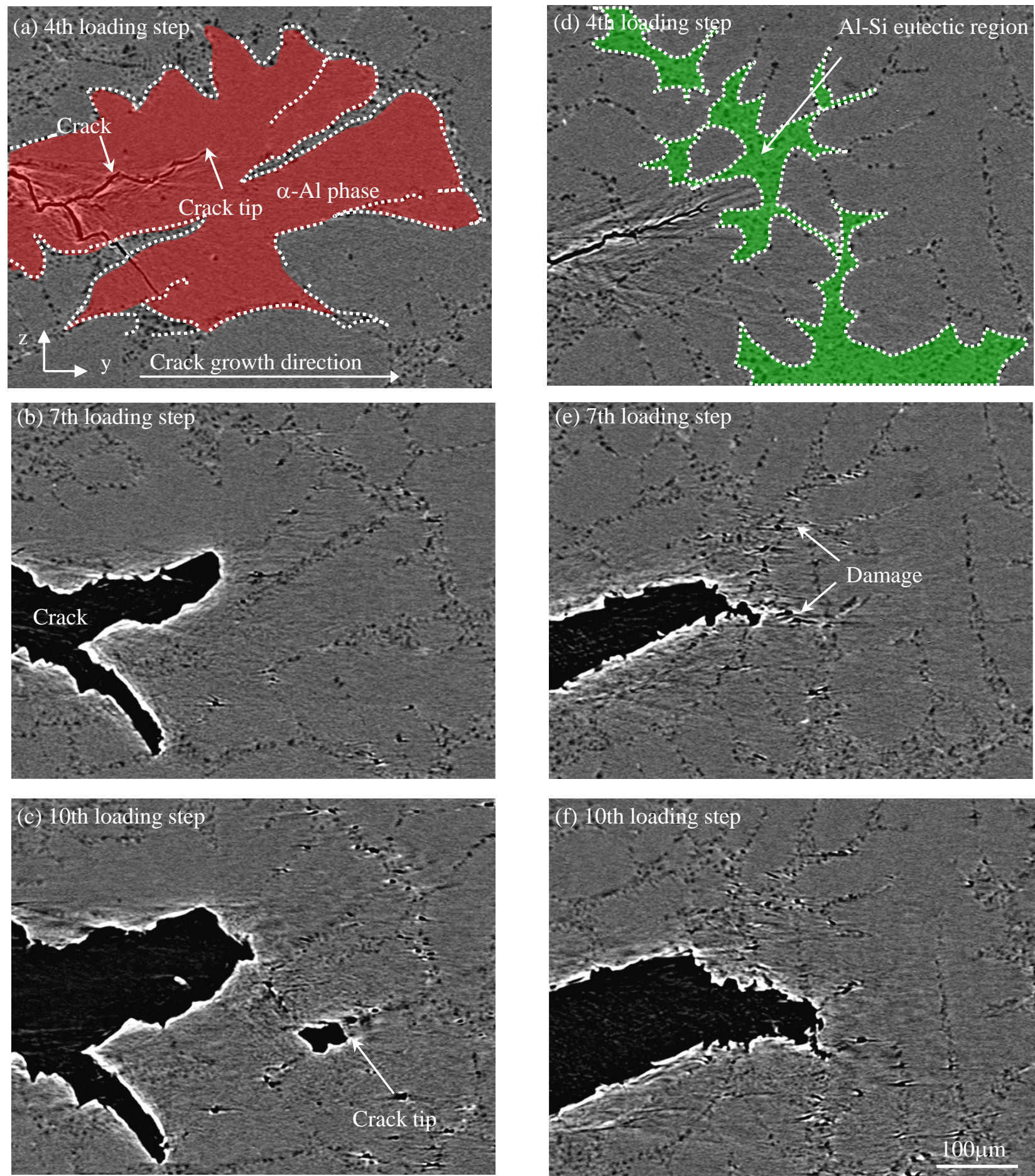

Crack tip located in an $\alpha-\mathrm{Al}$ phase

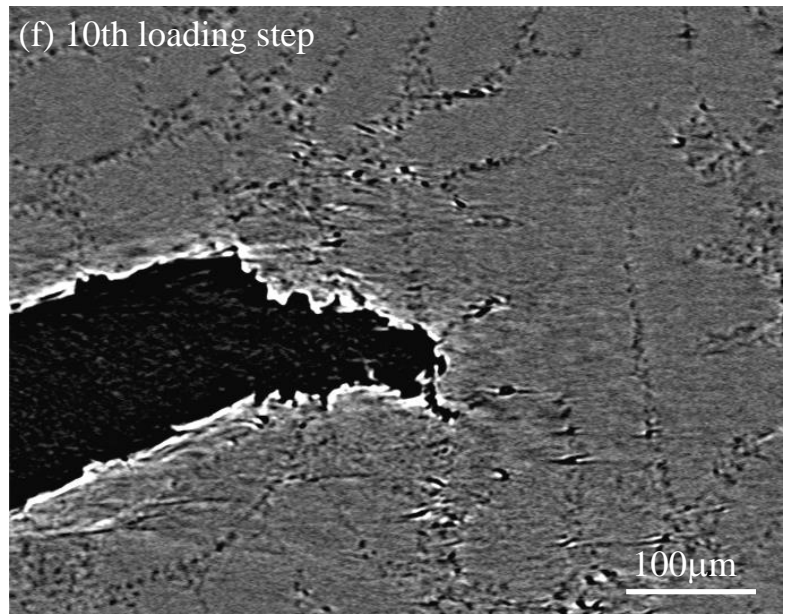

Crack tip located in an Al-Si eutectic phase

Fig.8 Tomographic slices showing the crack initiation and propagation in ductile $\alpha$-Al phase in (a) - (c) and a rather brittle Al-Si eutectic phase in (d) - (f), which correspond to slice numbers 312 and 527 (Specified in Fig. 7), respectively. Note that in (c) a void-like region is seen ahead of the crack-tip, which is actually connected to the main crack. 


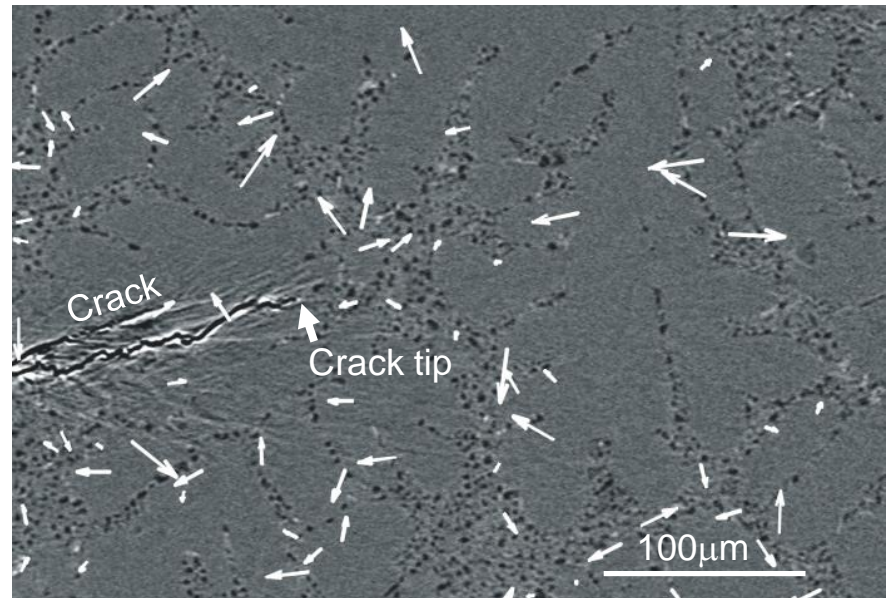

Crack growth direction

Fig.9 Result of the particle tracking indicating the amount and direction of particle displacement for each particle by a white arrow. 

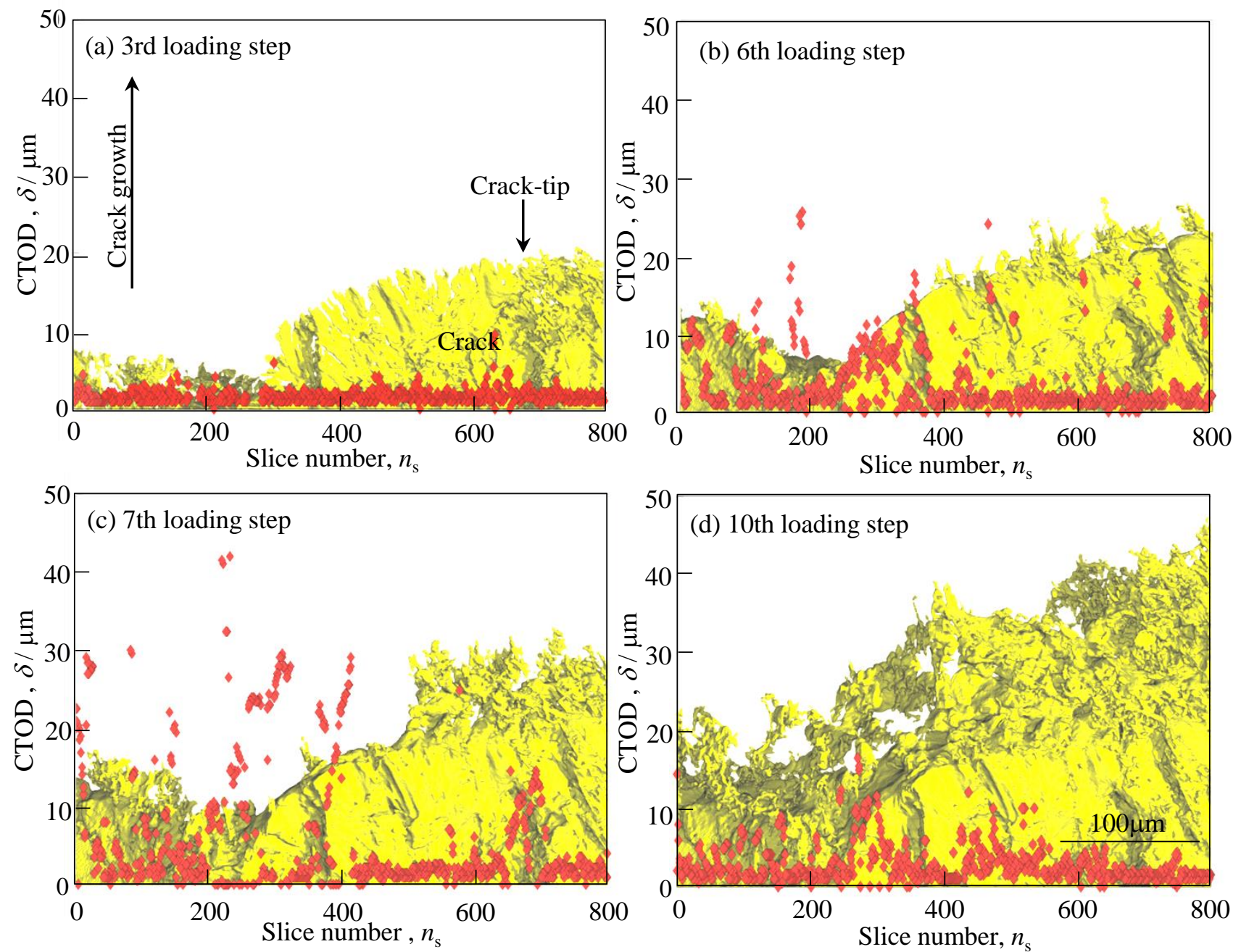

Fig.10 Comparison of the top views of the crack and the distributions of CTOD among different loading steps. 


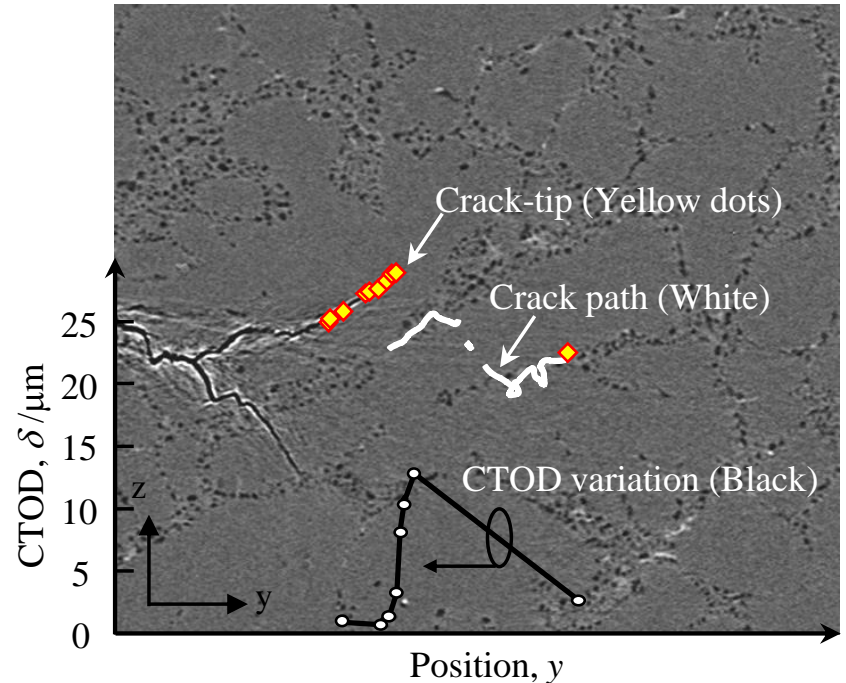

(a) Slice 320

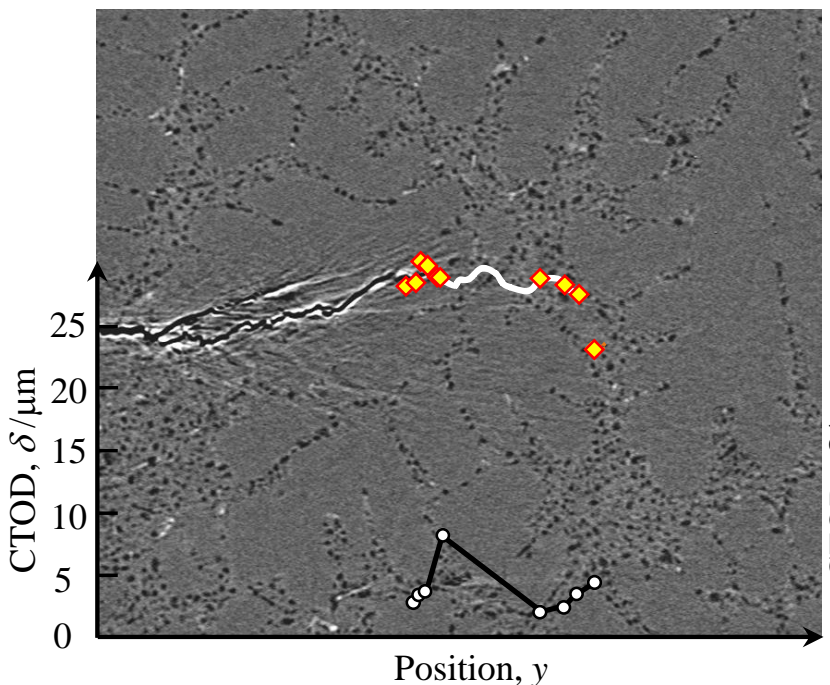

(c) Slice 500

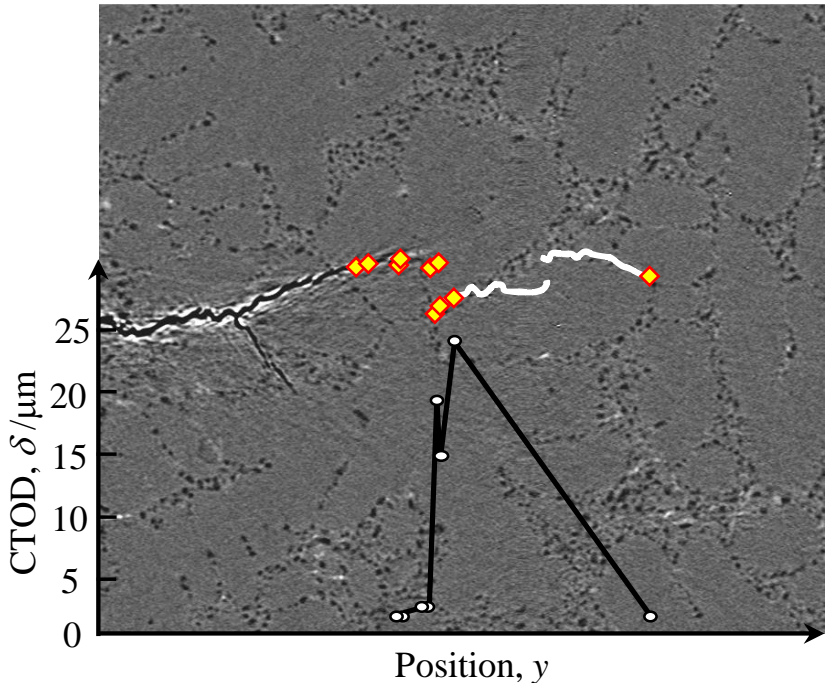

(b) Slice 400

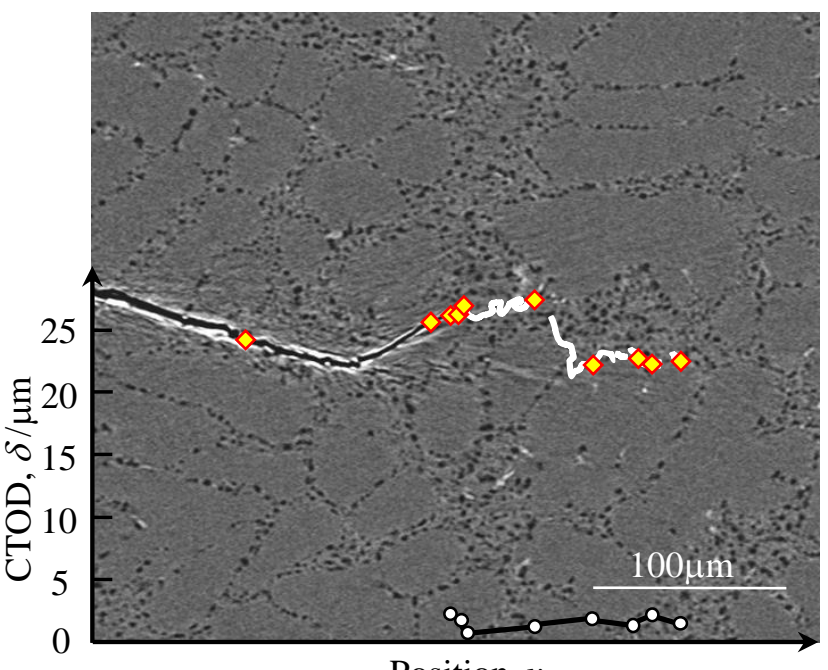

Position, $y$

(d) Slice 630

Fig.11 Tomographic slices showing the variations in crack tip location with crack blunting and propagation on four different slices together with CTOD variations. Note that the crack tip was initially located within an $\alpha$-Al phase in (a) and (b), in which uncracked lighaments were left after the final crack extension, while in (c) and (d) the crack tip was mainly tracing through an $\mathrm{Al}-\mathrm{Si}$ eutectic phase and more gradual and continuous crack extension was observed. 


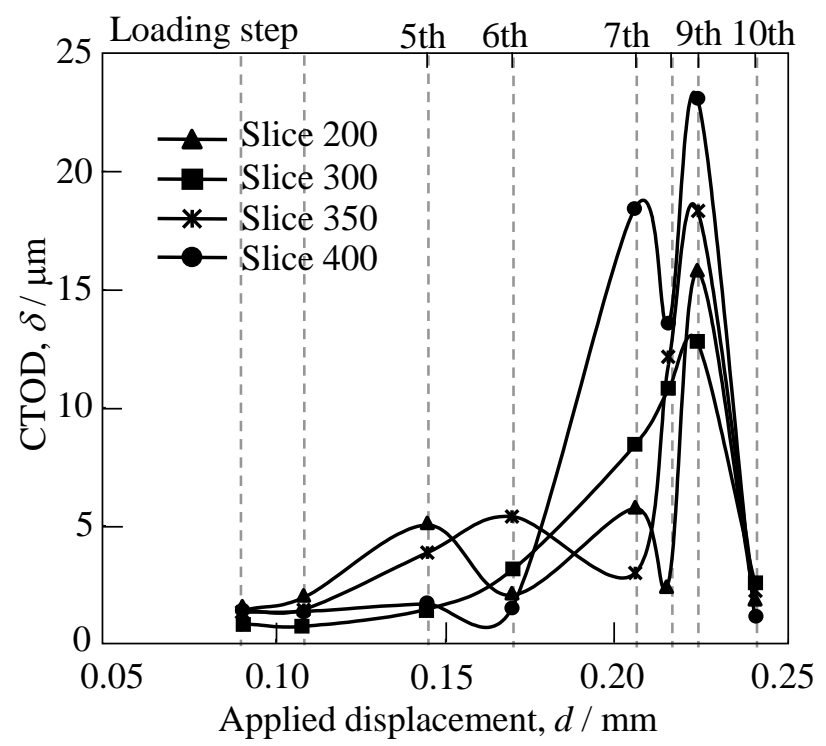

(a) The data for initial crack tip located in an $\alpha-\mathrm{Al}$ phase.

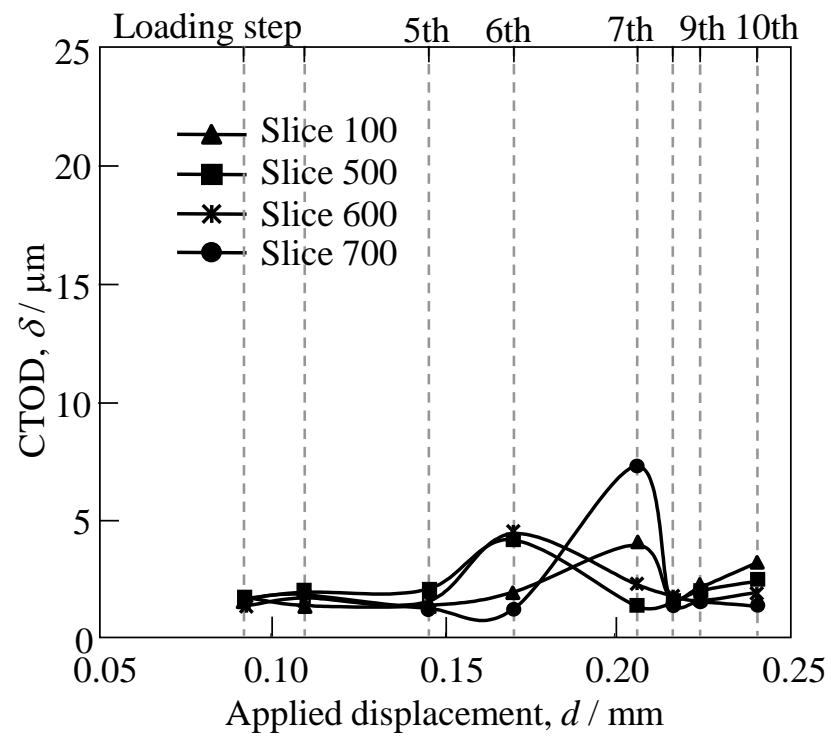

(b) The data for initial crack tip located in an Al-Si eutectic phase.

Fig. 12 Variations of CTOD as a function of applied displacement for different slices. (a) shows four examples for initial crack tip location in an $\alpha$-Al phase, while (b) shows those for initial crack tip location in an Al-Si eutectic phase. 


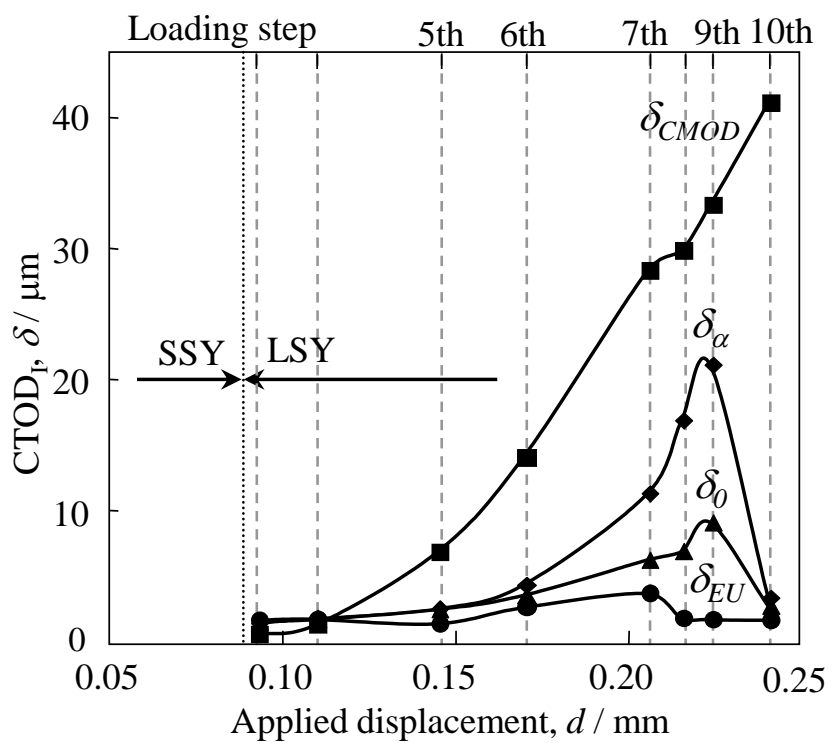

Fig.13 Comparison of the CTOD values obtained by the present $\left(\delta_{0}\right)$ and conventional macroscopic $\left(\delta_{\mathrm{CMOD}}\right)$ methods. $\delta_{\mathrm{CMOD}}$ is calculated based on a single CMOD value with assuming a virtual rotational center, while $\delta_{\alpha}$, $\delta_{\mathrm{EU}}$ and $\delta_{0}$ are the measured values averaged over the $\alpha$ $\mathrm{Al}$ and the Al-Si eutectic phases and the whole crack, respectively. 


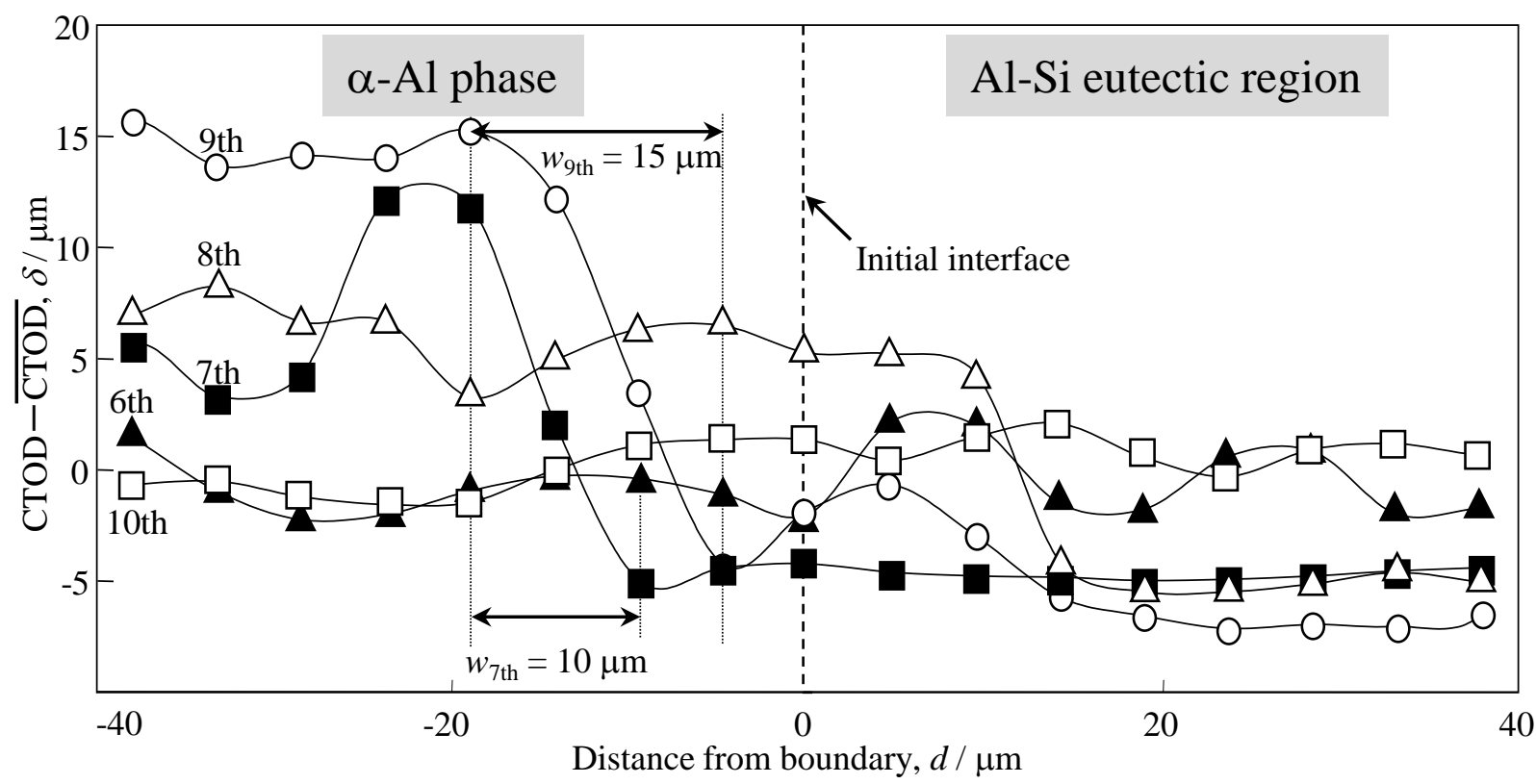

Fig. 14 CTOD variations across an interface between $\alpha$-Al and Al-Si eutectic phases at five different loading steps (i.e. the amount of crack extension). Obvious transitions can be identified for the 6th, 7th, 8th and 9th loading steps. The transition occurs over $15 \mu \mathrm{m}$ approximately at a maximum. $\overline{\mathrm{CTOD}}$ means the averaged value over the whole crack. 